\title{
Stop checking routine lipid panels every year
}

\author{
Some patients receive primary care from their gynecologist, including orders \\ for laboratory studies. This author argues that frequent routine lipid panels \\ are an unnecessary time and cost burden to patients and explains which \\ high-risk patients would benefit from such laboratory studies.
}

\section{Christopher Moriates, MD}

CASE 34-year-old woman with lipid panel results from 1 year ago

A woman with no chronic medical conditions was seen by her gynecologist for a routine wellwoman examination. She does not see another primary care provider. She is age 34 years and has a levonorgestrel intrauterine device that was placed after the birth of her second child 2 years prior. She does not take any other medications. She has never smoked and drinks a glass of wine with dinner a couple of times each week. She finds it challenging with her fulltime job and her parental responsibilities with 2 young children to get regular exercise but otherwise is active. She does not have a family history of premature cardiovascular disease. Last year, during her prior well-woman examination, she had a fasting lipid panel: her low-density lipoprotein (LDL) was $102 \mathrm{mg} / \mathrm{dL}$ (reference range, $\leq 160 \mathrm{mg} / \mathrm{dL}$ ), high-density lipoprotein (HDL) $52 \mathrm{mg} / \mathrm{dL}$ (reference range, $\geq 40 \mathrm{mg} / \mathrm{dL}$ ), triglycerides $140 \mathrm{mg} / \mathrm{dL}$ (reference range,

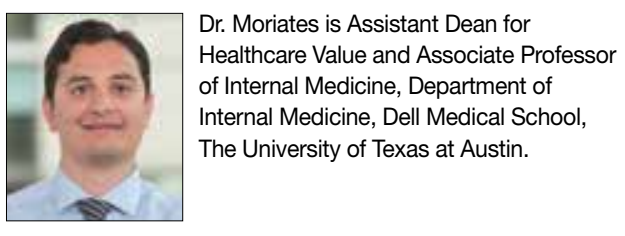

The author reports no financial relationships relevant to this article.

doi: 10.12788/obgm.0092
$<160 \mathrm{mg} / \mathrm{dL}$ ), and total cholesterol $182 \mathrm{mg} / \mathrm{dL}$ (reference range, $<200 \mathrm{mg} / \mathrm{dL}$ ).

During this visit, the patient's vitals are normal (blood pressure 116/58) and her physical examination is unremarkable. Her physician orders routine labs to be checked, including a fasting lipid panel. She has to figure out when she will be able to get these labs drawn, as she needs to coordinate with her work and childcare schedules. A week later, she leaves work at 4:00 PM and picks up her young children (aged 2 and 4 years) from childcare, bringing them to the laboratory to have her blood drawn. Not only are her children cranky in the waiting room, but she is feeling tired as she hasn't eaten all day because her physician told her she is supposed to be fasting. She has to pay for parking at the lot for the laboratory since it is connected to the medical center.

Was this lipid panel high value?

When and how often should we be checking lipid panels?

Do patients need to fast for these tests?

\section{The potential benefits and costs of routine lipid panel screening}

Hyperlipidemia is relatively prevalent, usually asymptomatic, and has been linked to cardiovascular outcomes. Thus, screening for lipid abnormalities is recommended to identify
IN THIS ARTICLE

Benefits and costs

this page

How often to perform screening

page 34

Is fasting required?

page 35 


\section{TABLE Summary of recommended approach}

\begin{tabular}{l} 
Consider obtaining baseline cholesterol screening for adult patients \\
\hline For adult patients at low cardiovascular risk and with normal lipid panel \\
screening results, consider initiating retesting at age 35 for men and age 45 \\
for women \\
\hline Most healthy adults should have repeat lipid panel testing every 4 to 6 \\
years \\
\hline Lipid panel tests may be performed non-fasting in most patients \\
Do not obtain expanded lipid panels (particle sizing, nuclear magnetic \\
resonance) as screening tests
\end{tabular}

FAST

TRACK

Like most services

in health care, the answer to whether or not a lipid panel is high cost is: it depends patients that would benefit from various interventions aimed at reducing cardiovascular disease risk, including lipid-lowering therapy. ${ }^{1}$ High levels of LDL cholesterol and low levels of HDL cholesterol are important risk factors for coronary heart disease.

Lipid panels are widely available blood tests with modest monetary costs, generally ranging from about $\$ 10$ to $\$ 100$ in the outpatient setting. Of note, a 2014 study examining inpatient charges for this common laboratory test found that hospital charges in California ranged from about $\$ 10$ to $\$ 10,000$ for a lipid panel. ${ }^{2}$ Despite the relatively low cost of each individual lipid panel, the aggregate costs to the health system of these frequently and widely performed tests are large. In fact, low-cost, high-volume health services, such as repeat cholesterol testing, account for the majority of unnecessary health spending in the United States, contributing nearly twice as much unnecessary cost as highpriced low-value services. ${ }^{3}$

To the patient, the cost is not only monetary. Some patients will need to take an additional hour or two off work as well as consider childcare, transportation, parking, and other mundane logistics to sit in a laboratory waiting room - a cost that may not be considered modest at all by the patient. ${ }^{4,5}$

Therefore, like most services in health care, the answer to whether or not a lipid panel is high-value care is: it depends. ${ }^{5}$ In the correct circumstances, the test generally is regarded as high value due to well-documented potential benefits and low monetary costs. However, when performed unnecessarily-either in patient groups that are unlikely to benefit or at intervals that are too soon to add helpful information-then all that is left are the financial and psychosocial costs, which make this a low-value test in these scenarios. For this patient, this test contributed to inconvenience and mild hardships with essentially no benefit, thus would be considered low-value care.

\section{When should we perform lipid screening in low-risk women?}

There are conflicting guidelines and opinions about at what age lipid screening should be routinely performed in adults. The United States Preventive Services Task Force (USPSTF) 2016 guidelines found "insufficient evidence that screening for dyslipidemia before age 40 years has an effect on either short- or longer-term cardiovascular outcomes." 6 Therefore, the USPSTF "recommends neither for nor against screening for dyslipidemia in this age group," and instead encourages "clinicians to use their clinical judgment for [these] patients." ${ }^{6}$

A common practice is to obtain a baseline lipid profile at the time of initiation of care with an adult primary care practitioner, if the patient was not previously screened, and to then determine subsequent testing based on these results and the patient's risk factors for cardiovascular disease. For patients with normal lipid screening results and lower cardiovascular risk factors (no hypertension, diabetes mellitus, cigarette smoking, family history of premature coronary heart disease), experts suggest follow-up lipid screening be performed in men at age 35 and in women at age $45 .^{7}$ Therefore, for this patient who had essentially a normal lipid panel a year prior, she should not have required repeat lipid testing until she is age 45 .

As for how frequently subsequent lipid testing should be performed, the Centers for Disease Control and Prevention states, "most healthy adults should have their cholesterol checked every 4 to 6 years." 8 Those taking lipid-lowering medications or those with risk factors such as heart disease, diabetes, or concerning family history should have their cholesterol checked more frequently. If 
patients are near a threshold for treatment, some experts suggest repeating measurements every 3 years, but even in these settings, annual testing would be considered excessive. $^{7}$

A standard lipid panel screen includes total cholesterol, LDL, HDL, and triglycerides. While a variety of assays have been developed that subfractionate lipoprotein particles based on size, density, or charge, these tests do not add value for low-risk patient screening and should only be used on an individualized basis for selected intermediate to high-risk patients. The American Society for Clinical Pathology released a Choosing Wisely recommendation that advises, "Do not routinely order expanded lipid panels (particle sizing, nuclear magnetic resonance) as screening tests for cardiovascular disease." $^{9}$

\section{Do lipid panels need to be fasting?}

For adults who are not taking lipid-lowering therapy, measurement of either a fasting or a nonfasting plasma lipid profile is effective for documenting baseline LDL and estimating cardiovascular risk. ${ }^{1}$ In other words, nonfasting lipid testing is appropriate for most lowrisk screening. Nonfasting testing generally is more convenient for patients; however, nonfasting lipid panels could result in elevated triglyceride levels. If an initial nonfasting lipid profile reveals a triglyceride level of $400 \mathrm{mg} / \mathrm{dL}$ or higher, then a repeat lipid profile in the fasting state should be performed for assessment of fasting triglyceride levels and baseline LDL. ${ }^{1}$ Some patients may prefer to simply get a fasting lipid panel initially so that they do not run the risk of having to return for a second test, especially if they are at increased risk for high triglyceride levels (ie, if they are obese, have diabetes, or are taking medications such as steroids, which can increase triglyceride levels).

\section{The bottom line}

Some patients receive primary care directly from their gynecologist, and thus it is important for women's health clinicians to be aware of appropriate cholesterol screening practices. While lipid panels may commonly be ordered routinely as part of annual health check-ups, the evidence suggests that this is an unnecessary practice that contributes to wasteful health spending at both individual and system levels; it also is an avoidable inconvenience for patients. It is unclear when lipid screening should be initiated for adult patients, but it seems reasonable to check baseline levels for a new patient who has not previously been screened. In lowrisk patients with normal lipid panel levels, experts recommend initiating retesting at age 45 for women and obtaining repeat lipid levels no more than every 4 to 6 years. For most patients, nonfasting lipid levels will suffice for screening. Avoiding common unnecessary testing is an effective way to improve value for patients.
References

1. Grundy SM, Stone NJ, Bailey AL, et al. 2018 AHA/ACC/ AACVPR/AAPA/ABC/ACPM/ADA/AGS/APhA/ASPC/NLA/ PCNA guideline on the management of blood cholesterol: executive summary: a report of the American College of Cardiology/American Heart Association Task Force on Clinical Practice Guidelines. J Am Coll Cardiol. 2019;73:31683209.

2. Hsia RY, Akosa Antwi Y, Nath JB, et al. Variation in charges for 10 common blood tests in California hospitals: a crosssectional analysis. BMJ Open. 2014;4:E005482.

3. Mafi JN, Russell K, Bortz BA, et al. Low-cost, high-volume health services contribute the most to unnecessary health spending. Health Aff. 2017;36:1701-1704.

4. Covinsky KE. The problem of overuse. JAMA Intern Med. 2013;173:1446.

5. Moriates C, Arora V, Shah N. Understanding Value-Based Healthcare. McGraw-Hill; 2015.
6. Chou R, Dana T, Blazina I, et al. Statins for prevention of cardiovascular disease in adults: evidence report and systematic review for the US Preventive Services Task Force. JAMA. 2016;316:2008

7. Vijan S. Screening for lipid disorders in adults. UpToDate website. Updated February 28, 2020. Accessed April 9, 2021. https://www.uptodate.com/contents/screening-for-lipiddisorders-in-adults

8. Getting your cholesterol checked. Centers for Disease Control and Prevention. Published September 8, 2020. Accessed April 9, 2021. https://www.cdc.gov/cholesterol/ cholesterol_screening.htm

9. American Society for Clinical Pathology. Choosing Wisely website. Published September 14, 2016. Accessed April 9 , 2021. https://www.choosingwisely.org/clinician-lists/ american-society-clinical-pathology-expanded-lipid -panels-to-screen-for-cardiovascular-disease

\section{FAST TRACK \\ Although nonfasting lipid testing can result in elevated triglyceride levels, it is appropriate for most low-risk screening}

